\title{
SPECTROSCOPIC AND KINETIC INVESTIGATIONS OF CONJUGATED BIRADICAL INTERMEDIATES
}

\author{
Jakob Wirz \\ Physikalisch-chemisches Institut der Universität Basel, \\ Klingelbergstrasse 80, $\mathrm{CH}-4056$ Base1, Switzerland
}

\begin{abstract}
Several reactive biradical intermediates of photochemical reactions were monitored directly in solution by the technique of kinetic and time-resolved spectrographic laser flash photolysis, and identified with the aid of low-temperature epr and optical spectroscopy, semiempirical calculations, as well as conventional steady-state analyses of product distributions. The focus of this report is on a number of mechanistic questions particular to the behaviour of biradicals and the experimental response obtained with a few selected mode1 systems. Some of the results may be expected to hold as guidelines and/or diagnostic tools of some general value in the field of biradical chemistry.
\end{abstract}

What is a Biradical (oid)?

I vividly remember several lively discussions on biradicals which were held at the third IUPAC Symposium on Photochemistry fourteen years ago when I joined the community as a newborn chemist. The chairman noted in his preface to the conference report: "One of these [informal discussion groups] attempted, without any great success, to reach a common understanding about the use of the term 'diradical', but nevertheless it led to a valuable exchange of ideas." ${ }^{l}$ It was precisely this confusion of ill-defined words and widely differing views, coupled with a general agreement about the key rôle of these will-o'-the-wisps in photochemistry, which has attracted me and probably many others. Shortly after this conference, Salem and Rowland have removed many of the basic misconceptions in their classical 'mise-au-point' on the electronic structure of biradicals (= diradicals) from the point of view of quantum theory, $^{2}$ and Michl has published a lucid and inspiring exposure of his qualitative ideas concerning the formation of biradicals in photochemical reactions. ${ }^{3}$ Some of the semantic discussions are continuing to date. I shall adhere to a pragmatic definition of a biradical as a molecular entitif whose lowest singlet and triplet state energies do not differ by much more than $\mathrm{kT}$, saij $10 \mathrm{~kJ} / \mathrm{mol}$. The expression "biradicaloid" would then extend this range to say $100 \mathrm{~kJ} / \mathrm{mol}$. This definition consciously does not make any statement about kinetic stability. In the MO description, biradicaloids have two approximately degenerate orbitals occupied by a total of two electrons. Normally the corresponding resonance structures have one bond less than requested by the usual rules of valence, but cyclobutadiene, molecular oxygien, and many ions provide exceptions to this staternent. 


\section{What Kinds of Photoreactions Proceed via Biralicals?}

In an article entitled "A Classification of Photochemical Reactions" Dauben, Salem and Turro have stated: "The underlying assumption in our classification is that all photochemical processes are controlled by generation of primary products which have the characteristics of diradicals." 4 Indeed the electronic excitation of a molecule by the absorption of a photon may be viewed as the separation of an electron pair and thus as a kind of bond-breaking process. The ubiquity of biradicals as primary photoproducts arises from the fact that biradicaloid geometries, which represent high-energy regions on the ground state hypersurface, usually correspond to minima on the lowest, electronically excited $\mathrm{S}_{1}$ and $\mathrm{T}_{1}$ surfaces (avoided crossings). These regions act as funnels ${ }^{3}$ which allow for a rapid conversion of the electronically excited molecule to a highly distorted species on the ground state surface. The above statement then implies that all photochemical processes are diabatic, i.e. the photoproducts are always formed in the electronic ground state via a biradical. This is obviously not quite true although it may be considered as a useful rule-ofthumb. An excellent review on adiabatic photoreactions has been compiled by Turro et al. 5
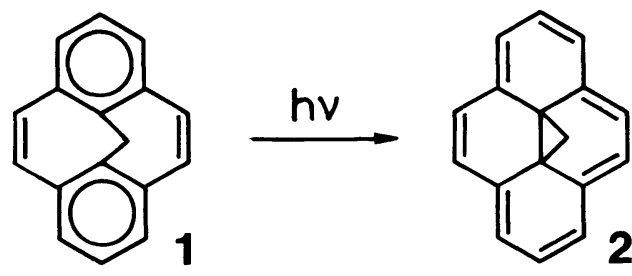

Let me briefly digress from the topic of my talk in order to present a prototype case for an adiabatic, electrocyclic ring closure reaction which proceeds entirely on the excited state surface, i.e. a pericyclic photoreaction which does not involve biradicals: the predominant primary photochemical process following electronic excitation of 8,16-methano[2.2]metacyclophane-1,9diene (l) is a rapid adiabatic valence isomerization to the excited singlet state $S_{1}$ of 10b,10c-methano-cis-10b,10c-dihydropyrene (2).6 A major piece of evidence was the observation of a red fluorescence emission ( $\lambda_{\max } 595 \mathrm{~nm}$ ) upon irradiation of colourless $\equiv$ (absorption onset ca. $380 \mathrm{~nm}$ ). Related examples of adiabatic cycloreversion reactions involving anthracenes have been presented two years ago at this symposium by Becker. 7 The driving force for the reaction $\underline{\underline{1}}^{*} \rightarrow \underline{\underline{2}}^{*}$ derives from the ca. $100 \mathrm{~kJ} / \mathrm{mol}$ aifference in the excitation energies of the stilbene-like chromophore $\stackrel{1}{=}$ and the bridged [14]annulene $\underline{\underline{2}}$ (Förster cycle). Obviously this thermodynamic criterion is a necessary but not a sufficient condition for the accurrence of an adiabatic reaction.

Due to obvious geometrical constraints the valence isomerization $\stackrel{\equiv}{\rightleftharpoons} \underline{2}$ is forced to follow a disrotatory pathway. Analysis of this reaction in terms of the Woodward-Hoffmann rules classifies it as "ground-state allowed", "excited-state forbidden", an extended version of the well known norcaradienecycloheptatriene isomerization. Note, however, that the orbital symmetry con- 
servation rules do not in general predict a symmetry imposed barrier for an excited state reaction, be it allowed or forbidden. In particular, no barrier is predicted from an orbital correlation diagram for $\stackrel{\equiv}{\rightleftharpoons}$ in either ground or excited state. The usual preference for "excited-state allowed" reactions derives from the fact that these proceed via the funnel associated with an avoided crossing. Thus, if a "forbidden" excited state reaction is feasible on energetic grounds, and if competitive "allowed" pathways are not available le.g. due to some stereochemical bias), the occurrence of an adiabatic reaction should be expected. More frequent examples will be encountered in proton transfer reactions which are not associated with avoided surface crossings. In essence, these predictions were made many years ago by Dougherty. ${ }^{8}$ Since a funnel on a triplet surface will be less effective due to the spin conservation rule, adiabatic reactions in the triplet manifold may occur quite frequently, but these will be more difficult to identify.

How Can Biradicals Be Detected and Identified?

A brief outline of the methods used in our laboratory is in order. Obviously, this choice does not include many important techniques such as timeresolved esr and Raman spectroscopy, or chemically induced dynamic nuclear polarization (CIDNP). Our main tool to monitor biradicals under laboratory conditions comparable to preparative work is flash photolysis, using a Qswitched Nd-laser as an excitation source to study reactions in the time domain from $10 \mathrm{~ns}$ to $100 \mu \mathrm{s}$, and a conventional electric discharge from $10 \mu \mathrm{s}$ upwards. 'These techniques, introduced by porter, 9 have become very popular and it is nowadays a relatively simple matter to obtain precise kinetic data on very short-lived transient intermediates. The difficulty obviously lies in the identification of these transients. More often than not, assignments based on comparisons of their absorption spectra with those of reference compounds or semiempirical calculations are inconclusive. Important clues may be derived from the response of these transients to changes of manageable parameters such as temperature, pressure, viscosity, solvent polarity, and added reagents such as oxygen. In our experience, it is absolutely essential to corroborate such tentative assignments by independent evidence.

First, both the qualitative and quantitative analysis of product distributions is a must. The quantitative data include quantum yield determinations and steady-state analyses of quenching and/or trapping processes (Stern-Volmer kinetics). The necessary consistency of the real-time and steady-state kinetic data provides a stringent test to mechanistic hypotheses. A second important method is the irradiation of glassy solutions at $77 \mathrm{~K}$ or of matrixisolated species at even lower temperatures. Quite frequently, the highly reactive primary photoproducts are still formed, but are stable indefinitely under these conditions. Obviously excited states, which may appear as transient intermediates in flash photolysis, will not survive for more than a few seconds and are thus discriminated. The persistent primary photoproducts can then be characterized by conventional spectroscopic methods. Of these, esr spectroscopy is of particular importance, since it can provide unambiguous 
evidence for the presence of biradicals in a triplet spin state.

A special feature of our flash photolysis setup is worth noting: the monitoring system allows for the simultaneous, single-shot detection of both the kinetics (at a given wavelength) and the transient spectrum (at a given time delay) in digital form with ns time-resolution. The spectral information is collected by a diode array camera. Essential to the use of this device in the ns time domain is a gateable microchannel image intensifier which not only enhances the signal intensity, but also acts as a very effective shutter with a risetime of ca. $2 \mathrm{~ns}$ and an on: off ratio $>10^{6}$. Thus the time delay after the flash and the time window to collect the transient spectrum can be chosen in any range down to a few ns. Thanks to the effective elimination of stray light this very sensitive setup is also useful to detect weak phosphorescence emissions from fluid solutions.

Let us now look at some examples of biradicals and biradicaloids which were studied by these direct methods. Lengthy arguments to justify our assignments shall be avoided and are deferred to recent or forthcoming original publications. Rather, some questions particular to the behaviour of biradicaloids will be discussed and an attempt will be made to draw some generalizable conclusions. We have chosen conjugated model systems for two reasons. First, they are suited for our predominantly optical methods of detection. Second, the delocalization of the radical sites and the concomitant introduction of structural constraints should endow such species with some kinetic stability towards an intramolecular collapse to stable isomers. Needless to say that others have made important contributions to the field, particularly in recent years. For key references see 10-16.

Can S- and T-State Biradicals be Distinguished on the Basis of

\section{Absorption spectra?}

Since esr spectroscopy can detect only triplet biradicals, there is need for a gool criterion to identify singlet biradicals. Consider the electronic absorption spectra of perinaphthadiyl biradicals ${ }^{17-19}$ (e.g. $\underline{3}^{\text {, }} \stackrel{4}{=}$ which are all very similar and are known ${ }^{18-20}$ to arise from T-T transitions. T-T spectra can be predicted with useful accuracy by PPP-SCI calculations. 21 It was shown experimentally ${ }^{18}$ that the first, highly structured absorption band near $500 \mathrm{~nm}$ is composed of two nearly degenerate, parity-forbidden electronic transitions of opposite polarization and this is predicted ${ }^{21}$ to be a general feature of alternant hydrocarbon biralicals. What would be expected for the unknown S-S absorption spectrum? Quantum theory tells us ${ }^{2}$ that biradicals will have three low-lying singlet states which would be degenerate in the absence of electronic interaction. Thus, a biradical in the lowest singlet state should exhibit two $S-S$ transitions at much longer wavelengths tinan the first $T-T$ transition. The replacement of the saturated alkyl bridge in $\underline{\underline{3}}$ by oxygen ( $\underline{\underline{5}}$ ) or nitrogen ( $\underline{\underline{6}}$ ) gives rise to a splitting of the nonbonding orbitals and these ylids have a singlet ground state. 22 Corresponaingly, their first absorption band lies at much longer wavelength (965 and $760 \mathrm{~nm}$, respectively) than that of triplet $\underline{3}$. The first absorption band of singlet $\underline{3}$, in which the two NBMO's are nearly degenerate, 
should therefore be well above $1000 \mathrm{~nm}$ in the near infrared (nir).<smiles>CC1(C)Cc2cccc3cccc(c23)C1</smiles><smiles>C[C@@H]1CCC[C@@H]2Cc3cccc4cccc(c34)[C@H]12</smiles><smiles>CC12C=CC=Cc3cccc(c31)C=[O+]C2</smiles><smiles>C[N+]1=Cc2cccc3cccc(c23)C[N+]1=O</smiles>

Since electronic transitions in the nir may be buried by solvent overtones or beyond the instrumental range, the absence of such a band can hardy be adduced as positive evidence for the assignment of a triplet biradical. ESR spectroscopy will do this job. On the other hand, if such a band is detected well beyond the predicted T-T transitions, a singlet biradical is at least a likely candidate. Good examples are provided by the singlet biradicals $\underline{=}$ and $\underline{\underline{8}}$, primary photoproducts of the natural quinones vitamin $\mathrm{K}$ and plastoquinone, which exhibit broad nir absorptions beginning at 2000 and $1600 \mathrm{~nm}$, respectively. 23 For comparison, the absorption onset of the blue singlet biradicaloid $\stackrel{9}{=}$ lies at $700 \mathrm{~nm} .^{24}$

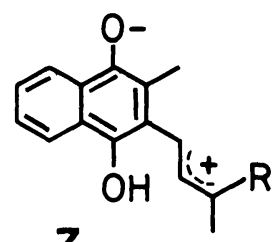

7<smiles>Cc1cc(O)c2ccccc2c1</smiles>

11
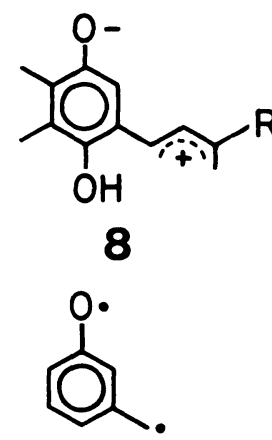

10<smiles>C=c1cccc2c1=C(O)C=CC2=O</smiles>

9

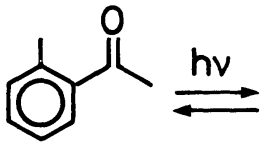<smiles>C=c1ccccc1=C(C)O</smiles>

12

Singlet biradicals should be highly polarizable due to the possibility of mixing three states of similar energy but widely different electronic distribution upon perturbation. Thus singlet biradicals respond very strongly to solvent polarity or substituent effects (kinetic and thermodynamic stability, $S_{0}-S_{1}$ transition energy). I will now discuss observations of such effects with the quinone methides $\underline{\underline{7}}$ to $\stackrel{9}{\underline{9}}$. The rate of intramolecular ketonization, which limits the lifetime of $\underline{\underline{9}}$, varies from $10^{5} \mathrm{~s}^{-1}$ in alkane solvents to $10 \mathrm{~s}^{-1}$ in polar aprotic solvents at room temperature. The first absorption band of $\underline{7}$ or $\underline{\underline{8}}$, prepared by photolysis of the quinone precursors in a rigid methyltetrahydrofuran glass at $77 \mathrm{~K}$, is blue-shifted by up to $300 \mathrm{~nm}$ when the glass is briefly tempered at ca. $80 \mathrm{~K}$. This is attributed to "solvation", i.e. to a readjustment of the solvent molecules when the glass softens slightly.

The preferential thermodynamic stabilization of the polarizable singlet state by polar substituents and/or solvents can be demonstrated with $\underline{\underline{7}}$ and $\underline{\underline{8}}$. 
A closer examination has revealed that the lowest $\mathrm{S}$ - and T-states happen to be in thermal equilibrium in these biradicals, $\mathrm{S}$ slightly below $\mathrm{T}$ in $\underline{\underline{7}}$ and vice versa in $\stackrel{3}{=}$. Interestingly, the parent 1,3-quinone methide (1으) and 1,3-

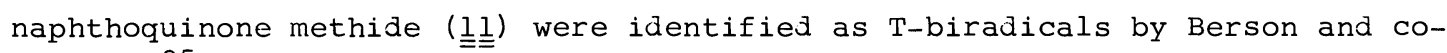
workers, 25 but a higher energy s-state was implicated as a reactive intermeliate in trapping reactions. Presumably, the additional hydroxyl substituent is mainly responsible for the relative stabilization of the $S-s t a t e$ in $\underline{7}$ and

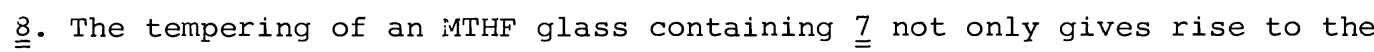
blueshift mentioned above, but also to a tenfold increase in the intensity of the nir band due to an increase in the $S$ : $T$ ratio induced by solvation. From the concomitant changes in the visible spectrum of $\underline{7}$, the first $\mathrm{T}-\mathrm{T}$ transition may be located at $660 \mathrm{nin}$. At room temperature, the same characteristic changes are observed in the transient uv/vis spectra of both $\underline{\underline{7}}$ and $\underline{\underline{8}}$; here, of course, the solvation process itself is beyond our time-resolution of 10 ns, but the equilibrium is clearly shifted towards the s-state in the more polar solvents.

\section{How Large is the Energy Gap Between the Lowest S- and T-States?}

The experimental determination of the energy gap $\Delta \mathrm{E}_{\mathrm{ST}}$ or of the equilibrium constant $\mathrm{K}_{\mathrm{ST}}$ is usually the most difficult problem and unless both the Sand T-biradical (oid) are metastable intermediates, these quantities do not even have a well-defined meaning. The absolute prediction of potential energy minima on the lowest $S-$ and T-surfaces and their relative energies by $a b$ initio calculations is also a demanding task. It is nevertheless a remarkable fact that for each of the prototype systems methylene, cyclobutadiene, and trimethylenemethane the "best" available experimental evilence was at one time in the last decade considered to be in serious conflict with the "best" theoretical preaictions, and that, in each case, it was the interpretation of these experiments which has since been shown to be in error. For larger biradicaloids, useful qualitative guidelines or semiempirical calculations are available for an a priori estimate of $\Delta \mathrm{E}_{\mathrm{ST}} \cdot 26$

For persistent biradicals equilibrium constants $K_{S T}$ near unity can be determined by esr spectroscopy. The spectra obtained with glassy solutions of randomly orientea biradicals, e.g. $\underline{\underline{7}}^{23}$ essentially yield three pieces of information. First, the characteristic shape of these spectra and the presence of a signal at half-field provide unambiguous proof for the existence of a persistent triplet species in the sample. Second, the width of the spectrum, characterized by the zero-field parameters $D$ and $E$, gives a rough indication about the average distance of the unpaired electrons and the shape of the triplet species. Detailed structural information is not available, since hyperfine splittings are usually not resolved. Double resonance experiments (ENDOR) may improve this situation. 27 Third, the signal intensity dependence on temperature can be used to determine $\mathrm{K}_{\mathrm{ST}}$.

ESR signal intensities are inversely proportional to the temperature $\mathrm{T}$ (Curie's law) due to the near-equal Boltzmann population of the spin sublevels at temperatures above a few K. Deviations from Curie's law will be ob- 
served, if other processes are involved such as the thermal equilibration between the lowest $\mathrm{S}$ - and T-states of a biradical. Due care must be taken to avoid artefacts such as the irreversible depletion of the sample at elevated temperature an $₫$ saturation effects at low temperature. ${ }^{18}$ strictly, the adherence to Curie's law can mean either of two things: $\mathrm{K}_{\mathrm{ST}}=3\left(\Delta \mathrm{E}_{\mathrm{ST}}=0\right)$ or $\mathrm{K}_{\mathrm{ST}} \gg 3$ ( $\mathrm{T}$ ground state). In practice, good experimental data are consistent with the first case only within extremely narrow limits, e.g. $\left|\Delta \mathrm{E}_{\mathrm{ST}}\right|<0.03$ $\mathrm{kJ} / \mathrm{mol}$. Thus, if a biradical obeys Curie's law in several media of different polarity, then the triplet state may be identified as the ground state, i.e. the first possibility may be safely discarded due to the widely different polarizabilities of Sand T-biradicals. The same argument applies, if several derivatives of a biradical obey Curie's law, such as 1,8-naphthoquinodimethane and its alkylbridged derivatives. $18-20$

As would be anticipated from the absorption data on $\stackrel{7}{=}$, preliminary experiments do indicate non-Curie behaviour of this biralical, particularly in polar media. ${ }^{23}$ Unfortunately the esr method to determine $\Delta \mathrm{E}_{\mathrm{ST}}$ is restricted to a very narrow range of values, especially for reactive species which are persistent only at low temperatures. Most biradicaloids will either give no detectable esr signals (S ground state, e.g. the limits of error ( $T$ ground state).

Another method to determine $\Delta \mathrm{E}_{\mathrm{ST}}$ values of singlet ground state biradicaloids consists of measuring triplet energy transfer rates in fluid solution by kinetic flash photolysis, using a graded series of sensitizers. We have used this method to estimate the triplet energy of a peralkylated cyclobutadiene derivative, $\Delta \mathrm{E}_{\mathrm{ST}}=50 \pm 5 \mathrm{~kJ} / \mathrm{mol}, 28$ and of an alkyl-bridged o-quinodimethane, $\Delta \mathrm{E}_{\mathrm{ST}}=110 \pm 10 \mathrm{~kJ} / \mathrm{mol} .^{29}$ We are presently seeking to close the gap between the two methods (low temperature esr for $\left|\Delta \mathrm{E}_{\mathrm{ST}}\right| \leqslant 2 \mathrm{~kJ} / \mathrm{mol}$; energy transfer for $\left.\left|\Delta \mathrm{E}_{\mathrm{ST}}\right| \gtrsim 50 \mathrm{~kJ} / \mathrm{mol}\right)$ by using Chichibabin-type hydrocarbons as sensitizers. For these isolable, but air-sensitive compounds, Brauer and Reinsch have determined $\Delta \mathrm{E}_{\mathrm{ST}}$ values covering the range from 4 to $50 \mathrm{~kJ} / \mathrm{mole}$ using the esr method at elevated temperatures. 30

The decay of triplet ground state biradicaloids usually involves intersystem crossing to the singlet in the rate-determining step; this is reflected in lower-than-usual preexponential factors, $A<10^{14} \mathrm{~s}^{-1}$, in the temperature dependence of the rate constant. It is then tempting to interpret the corresponding activation energies $\mathrm{E}_{\mathrm{a}}$ as a measure of $\Delta \mathrm{E}_{\mathrm{ST}}$. Unfortunately there is no real connection between the two quantities, as displayed below in terms of arbitrary hypersurfaces.
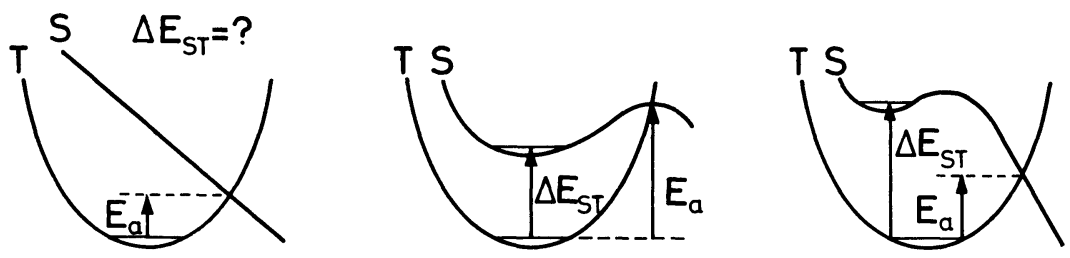
For a lack of direct physical methols to determine $\Delta \mathrm{E}_{\mathrm{ST}}$, chemists have often resorted to clever schemes aime $\mathfrak{l}$ at deriving $\mathrm{K}_{\mathrm{ST}}$ of biradicaloid intermediates from product distributions or kinetic studies of trapping reactions. Such analyses can yield quite precise results which, however, depend upon the correctness of some unproven assumption(s), e.g. the skell hypothesis for carbenes. Such an estimate for $\mathrm{K}_{\mathrm{ST}}(\underline{\underline{8}})$, derived from oxygen quenching rates, will be given below.

\section{Intersystem Crossing (ISC) at Biradicaloid Geometries}

The question of $S \rightarrow T$ and $T \rightarrow S$ ISC rates is central to any mechanism involving biradicals. As is well known from a wealth of photophysical studies of excited states, ISC rates can vary rather unpredictably over many orders of magnitude. Major trends can be rationalized in terms of perturbation theory for spin-orbit coupling and the energy gap law for radiationless transitions. As a rule-of-thumb for conjugated systems: the "spin barrier" reauces ISC rates by a factor of $10^{6}$ for $\pi \pi^{*}$ states, $10^{3}$ for $n \pi^{*}$ states, in comparison with related (same energy gap), spin-allowed radiationless conversions. Thus $\mathrm{k}_{\text {ISC }} \approx 10^{10} \mathrm{~s}^{-1}$ is likely to hold as an upper limit in the absence of heavy atoms and we may assume that the spin conservation rule is generally observed for single transitions across an S/T-surface intersection. Opposing views have been expressed, but I am not aware of a convincing counterexample. In short: S-biradicals are originall: formed from $S$-precursors, T-biradicals from $T$-precursors in photochemical reactions.

The lifetime of S-biradicals continues to be a matter of dispute, but recent experimental and theoretical estimates seem to converge to the opinion that the barriers predicted by Benson's thermochemical method, e.g. 20 to 40 $\mathrm{kJ} / \mathrm{mol}$ for trimethylene reacting to cyclopropane, are far too high. ${ }^{15}$ The spin barrier should be reflected in unusually low A-factors for ' 'ISC' whereas the collapse to stable products is expected to exhibit A-factors not much below $10^{-13} \mathrm{~s}^{-1}$. We conclude that ISC from a $S$-biradical is favoured at low temperature and is efficient only, if its lifetime exceeds $10^{-10} \mathrm{~s}$. Few examples are available to document this working hypothesis. ${ }^{10}$ we now have evidence that the ring closure yielding dimethylnaphthocyclopropane from $S-\underline{3}$ is far more efficient (> 99\%) than ISC to the $\mathrm{T}$ ground state of $\underline{\underline{3}}$ even at $77^{\mathrm{K}} \mathrm{K}{ }^{19}$ In contrast, ISC to $\mathrm{T}-\underline{\underline{4}}$ competes (ca. 10\%) with naphthocyclobutane formation from $\mathrm{S}-\underline{4}$ at room temperature and dominates at $77 \mathrm{~K}$. The lifetime of $\mathrm{S}$ - $\underline{\underline{4}}$ at room temperature is estimated as ca. $10^{-9} \mathrm{~s}$ on the basis of oxygen trapping reactions. 31

The decais of T-biradicals frequently involves ISC in the rate-determining step and can be monitored directly by flash photolysis or, at low temperature, by esr-spectroscopy. It should, however, be realized that the low-temperature data may well not extrapolate linearly for various reasons (tunnelling, matrix viscosity effects near the glass transition temperature, pathways with low $A$ - and $E_{a}$-values favourea at low temperature). Examples of spin prohibited decay rates observed in our laboratory include the triplet perinaphthadiyls, $\mathrm{k}(\underline{\underline{3}})=10^{8.4 \pm 0.2} \exp [-(3500 \pm 100) / \mathrm{T}] \mathrm{s}^{-1}, 19 \mathrm{k}(\underline{\underline{4}})=10^{6.9 \pm 0.3}$ $\exp [-(2300 \pm 200) / T], 18$ and the rate of ketonization of the photoenol $12 \underline{2}$ which, 
in apolar solvents, proceeds at least six times slower from the excited Tstate $\left(\Delta \mathrm{E}_{\mathrm{ST}}=-100 \mathrm{~kJ} / \mathrm{mol}\right)^{29,32}$ than from the ground s-state. 33 on the other hand, the opposite case was observed with the quinone methides $\underline{\underline{7}}$ and $\underline{\underline{8}}$ where the $\mathrm{S} / \mathrm{T}$ equilibrium is established rapidly (< $10 \mathrm{~ns})$, much faster than the decay to stable products which proceeds in the upper $\mu s$ range in the absence of trapping agents. 23

The ISC rates can be enhanced by the addition of heavy-atom solutes or paramagnetic reagents and such experiments provide an important diagnostic tool in biradical chemistry. As mentioned earlier, the interpretation of ${ }^{3} \mathrm{O}_{2}$ or ${ }^{2}$ NO quenching rates involves a number of assumptions ${ }^{34}$ which, however, are fairly well substantiated. With these assumptions, oxygen-quenching rates can be immediately predicted from spin-statistical factors for a given mechanistic scheme or, conversely, an unknown parameter of such a scheme, e.g. $\mathrm{K}_{\mathrm{ST}}$ ' can be evaluated from observed quenching rates. Some examples follow. The decay rate of $\mathrm{T}-\underline{\underline{4}}$ is increased sevenfold in the presence of $0.2 \mathrm{M}$ dimethylmercury in a degassed methanol solution at room temperature. ${ }^{18}$ It also increases linearly with increasing oxygen pressure; the bimolecular rate constant for oxygen quenching amounts to $\mathrm{k}_{\mathrm{q}}=(2.4 \pm 0.2) \cdot 10^{9} \mathrm{M}^{-1} \mathrm{~s}^{-1}, 35$ in excellent agreement with the prediction $k_{q}=k_{\text {dijf }} / 9$ for a triplet ground state biradicaloid $\left(\Delta E_{S T}>k T\right)$. We have observed in several instances that the reactions of $S$ ground state biradicaloids $\left(\Delta E_{S T}<-k T\right)$ are invariably several orders of magnitude below the limit of diffusion control. Examples include $\underline{\underline{5}}$, $\underline{\underline{6}}$, and $\underline{\underline{9}}$. Furthermore, the reaction rates are highly sensitive to solvent purity, solute concentrations, and added inhibitors; presumably these spin-forbidden autoxidations proceed by complex radical chain mechanisms. Note, however, that the oxygen quenching rates for $S$-biradicaloids with a $T$ ground state are expected to approach the diffusion-controlled limit. Finally, the oxygen quenching rates of the quinone methide 8 were found to decrease with increasing solvent polarity, ranging from $(3.1 \pm 0.4) \cdot 10^{8} \mathrm{M}^{-1} \mathrm{~s}^{-1}$ in isooctane to $(1.1 \pm 0.1) \cdot 10^{6}$ $\mathrm{M}^{-1} \mathrm{~s}^{-1}$ in actonitrile at room temperature. This is attributed to the stabilisation of $\mathrm{S}-\underline{\underline{8}}$ in polar solvents as discussed previously. With the assumption that one out of nine encounters between $\mathrm{T}-\underline{\underline{8}}$ and ${ }^{3} \mathrm{O}_{2}$ will quench the intermediate by spin-allowed hydroperoxide formation, encounters with $\mathrm{S}-\underline{\underline{8}}$ being ineffective, we can derive the equilibrium constant $\mathrm{K}_{\mathrm{ST}}=[\mathrm{T}] /[\mathrm{S}]$ as $8 \cdot 10^{-2}$ in isooctane and $5 \cdot 10^{-4}$ in acetonitrile. 23

\section{Photoreactions of Biradicaloid Intermediates}

Sequential biphotonic reactions arising through light absorption by biradicaloid intermediates may occur efficiently witn high-intensity light sources lflash photolysis, laser irradiations) and in low-temperature irradiations. This can give rise to quite unexpected changes in product distribution when the temperature or the light intensity are varied. Two examples for photoreactions of T-biradicals are shown in the scheme overleaf. 18,36

Singlet biradicaloits are less likely to be photoreactive, since their low-lying excited states will tend to make radiationless processes dominant (funnel effect). We have investigated several carbonyl ylids formed by photochemical ring opening of oxiranes 22,37 . In contrast to an earlier observation ${ }^{38}$ we 
have found that the carbene intermediates accompanying these reactions are formed directly from the excited oxirane by $\mathrm{C}-\mathrm{O}$ bond cleavage and not by secondary photolysis of the carbonyl ylids.
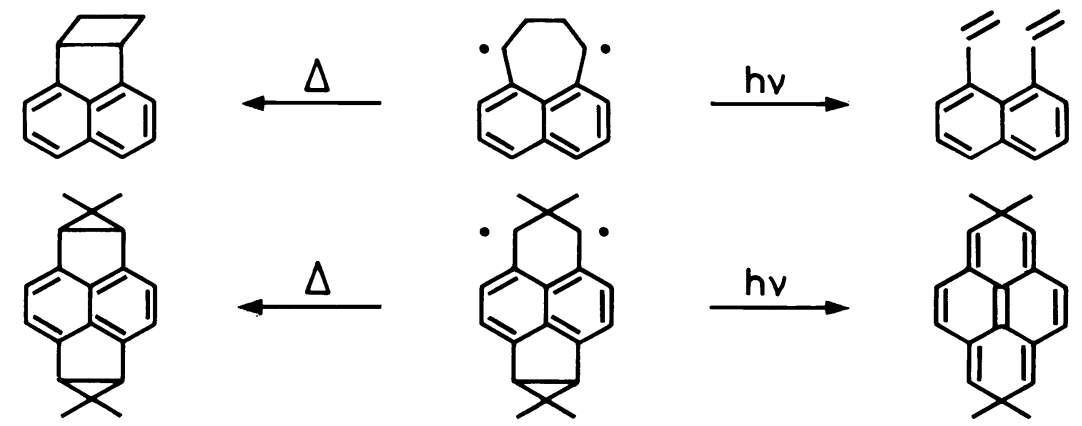

\section{So What?}

The ubiquity of biradicals in mechanistic schemes of organic chemistry contrasts with the paucity of experimental knowledge concerning these fleeting species. Also, the potential utility of biradicals as bifunctional reactants, capable of forming two new C-C bonds simultaneously, has so far been little exploited, 39 except, of course, with highly stabilized S-ground state biradicaloids such as azomethine ylids ${ }^{40}$ and orthoquinodimethane. ${ }^{41}$ The inherently complex and seemingly unpredictable behaviour of biradicals as reactive intermediates arises from the thermal accessibility of two or more electronic states with widely different properties. Once the key problems, the relative energy of the lowest $\mathrm{S}$ - and T-states and the rates of intersystem crossing, have been solved (or correctly guessed at), the chemical reactivity becomes interpretable and qualitatively predictable by "chemical intuition."

Flash photolysis is extremely useful to monitor directly the kinetics of reactive intermediates under laboratory conditions. A quick and efficient screening of solvents and reagents is possible to study structure-reactivity relationships or to choose optimal reaction conditions for synthetic purposes. 42 The key cyclization step in Quinkert's elegant stereoselective synthesis of estrone, the photoenolization of an ortho-methylphenyl ketone, was successful only under quite unusual ("seemingly alchimistic") reaction conditions. 43 These conditions were not found by serendipity, but by an extremely clever and deliberate exploitation of, inter alia, previous mechanistic investigations of the parent substrate 12.33 For obvious reasons, flash photolysis has been applied mainly to study photochemical reaction mechanisms. However, many reactive intermediates of prime interest to ground state chemistry can also be generated photochemically from suitable precursors and thus become easily accessible to real-time kinetic studies. E.g., the first absolute measurement of the acidity and keto-enol equilibrium constants of acetone in aqueous solution was recently obtained by photolytic generation of the enol through Norrish Type II cleavage of alkanones. ${ }^{4}$ I see here a wide field for future applications. 
This work is part of projects $\mathrm{nr} .2 .616-80$ and 2.470-82 of the Swiss National Science Foundation. Generous support by Ciba-Geigy SA, F. Hoffmann-La Roche \& Cie. SA, and Sandoz SA, Basel, is gratefully acknowledged.

\section{References}

1) D. Bryce-Smith, Pure Appl. Chem. 24 (3), p. VII (1970).

2) L. Salem, C. Rowland, Angew. Chem. 84, 86 (1972); Angew. Chem. Int. Ea. Engl. 11, 92 (1972); see also ref. 15 .

3) J. Michl, Mol. Photochem. 4, 243, 257, and 287 (1972).

4) W.G. Dauben, L. Salem, N.J. Turro, ACC. Chem. Res. 8, 41 (1975).

5) N.J. Turro, J. McVey, V. Ramamurthy, P. Lechtken, Añgew. Chem. 91, 597 (1979); Angew. Chem. Int. Ed. Engl. 18, 572 (1979).

6) J. Wirz, G. Persy, E. Rommel, I. Murata, K. Nakasuji, Helv. Chim. Acta $67,305(1984)$.

7) H.-D. Becker, Pure Appl. Chem. 54, 1589 (1982).

8) R.C. Dougherty, J. Am. Chem. Soc. 93, 7187 (1971).

9) G. Porter, M.A. West, Techniques of Chemistry Vol. VI, part II, 3rd edition (G.G. Hammes, ed.), Wiley-Interscience, New York 1979.

10) J.A. Berson and coworkers, J. Am. Chem. Soc. 104, 2209 seq. (1982); 105, 5144 (1983); 106, 1867 (1984).

11) R. Caldwell, plenary lecture at this symposium.

12) J. Michl (ed.), Tetrahedron (Symposium-in-print) 38, 735 seq. (1982).

13) W.R. Roth, B.P. Scholz, Chem. Ber. 115,1197 (1982).

14) J.C. Scaiano, Acc. Chem. Res. 15, $2 \overline{52}$ (1982).

15) W.T. Borden (ea.), "Diradicals", Wiley-Interscience, New York 1982.

16) P. de Mayo (ed.), "Rearrangements in Ground and Excited States (3 Vols.)", Academic Press, New York 1980.

17) J.-F. Muiller, D. Muller, H.J. Dewey, J. Michl, J. Am. Chem. Soc. 100, 1629 (1973).

18) M. Gisin, E. Rommel, J. Wirz, M.N. Burnett, R.M. Pagni, J. Am. Chem. Soc. 101,2216 (1979).

19) E. Hasler, E. Gassmann, J. Wirz, in preparation.

20) M.S. Platz, J. Am. Chem. Soc. 101, 3398 (1979).

21) M. Gisin, J. Wirz, Helv. Chim. Acta 66, 1556 (1983).

22) W. Leupin, P. Haspra, J. Wirz, contributed paper at this symposium (thesis of W.L., 1980; P.H., 1982).

23) M.A. Hangarter, J. Wirz, to be published (thesis of M.A.H., 1983).

24) E. Rommel, J. Wirz, Helv. Chim. Acta 60,38 (1977).

25) J.A. Berson and coworkers in ref. 12, page 787.

26) J. Koutecký, D. Döhnert, P.E.S. Wormer, J. Paldus, J. Cǐ̌ek, J. Chem. Phys. 80, 2244 (1984) and references therein; see also W. Leupin, J. Wirz, J. Am. Chem. Soc. 102, 6068 (1980).

27) B. Kirste, H. van Willigen, H. Kurreck, K. Möbius, M. Plato, R. Biehl, $\mathrm{J}$. Am. Chem. Soc. 100, 7505 (1978).

28) J. Wirz, A. Krebs, H. Schmalstieg, H. Angliker, Angew. Chem. 93,192 (1981); Angew. Chem. Int. Ed. Engl. 20, 192 (1981).

29) P. Forster, R. Gschwind, E. Haselbach, U. Klemm, J. Wirz, Nouv. J. Chim. 4, 365 (1980); M. Capponi, J. Wirz, unpublished.

30) H.-D. Brauer, E.-A. Reinsch, Ber. Bunsenges. 77, 348 (1973).

31) R.M. Pagni, J. Wirz, unpublished work.

32) C.V. Kumar, S.K. Chattopadhyay, P.K. Das, J. Am. Chem. Soc. 105 5143 (1983).

33) R. Haag, J. Wirz, P.J. Wagner, Helv. Chim. Acta 60, 2595 (1977).

34) The following assumptions are involved: (i) Catalyzed ISC by "spin exchange" (Dexter mechanism) occurs only in contact pairs. (ii) The total spin of an encounter complex is conserved during the encounter lifetime. (iii) The thermodynamic equilibrium for spin-allowed exchange processes is reached during a single encounter. (iv) Spin-allowed oxygen addition reactions ( $T+T$ "Diels-Alder" or "ene"-reactions) are efficient. For the use of spin-statistical factors see O.L.J. Gijzeman, F. Kaufman, G. Porter, J. Chem. Soc. Faraday Trans. II, 69, 708 (1973) and ref. 14.

35) The larger value reported earlier 18 was determined at very low $\mathrm{O}_{2}$ pressures and was since found to be in error.

36) J. Ackermann, H. Angliker, E. Hasler, J. Wirz, Angew. Chem. 94, 632 (1982); Angew. Chem. Int. Ed. Engl. 21, 618 (1982); Angew. Chem. Suppl. 1982,1429 . 
37) N. Bischofberger, B. Frei, J. Wirz, Helv. Chim. Acta 66, 2489 (1983).

38) G.W. Griffin, K. Ishakawa, I.J. Lev, J. Am. Chem. Soc. 98, 5697 (1976).

39) D.R. Little, G.W. Muller, J. Am. Chem. Soc. 103, 2744 (1981).

40) R. Huisgen, Angew. Chem. 89, 589 (1977); Angew. Chem. Int. Ed. Engl. 16, $572(1977)$.

41) J.J. MCCullough, Acc. Chem. Res. 13, 270 (1980); W. Oppolzer, Angew. Chem. 89, 10 (1977); Angew. Chem. Int. Ed. Engl. 16, 10 (1977).

42) P. Schiess, M. Eberle, M. Huys-Francotte, J. Wirz, Tetrahedron Lett. 1984,2201 .

43) G. Quinkert, H. Stark, Angew. Chem. 95, 651 (1983); Angew. Chem. Int. Ed. Engl. 22, 637 (1983).

44) Y. Chiang, A.J. Kresge, Y.S. Tang, J. Wirz, J. Am. Chem. Soc. 106, 460 (1984). 